\title{
Infliximab therapy in two cases of severe neurotuberculosis paradoxical reaction
}

\author{
there is \\ mounting \\ evidence \\ for the use \\ of tumour \\ necrosis \\ factor alpha \\ antagonists \\ such as \\ infliximab
}

James S Molton
MBBS, BSC, FRACP

Paul J Huggan

BSc(Hons), MBChB

\section{Sophia Archuleta}

MD

National University Health System Singapore.

sophia@nus.edu.sg

\section{Clinical record}

\section{Patient 1}

A 60-year-old HIV-negative woman presented with a week's history of fever, vomiting and confusion, followed by progressive personality change. On admission, she was noted to have urinary retention, left oculomotor nerve palsy and an upgoing right plantar response. A magnetic resonance image (MRI) of the brain showed leptomeningeal enhancement with gyral swelling and subtle cortical T2 signal hyperintensity in the right frontal lobe, suggesting meningoencephalitis. Cerebrospinal fluid (CSF) cultures grew fully susceptible Mycobacterium tuberculosis. Antituberculous therapy was started with isoniazid, rifampicin, ethambutol and pyrazinamide, plus dexamethasone. CSF cultures tested negative by Week 1 . Over the next month, she had ongoing fevers and fluctuating conscious state. High CSF pressures necessitated ventriculoperitoneal (VP) shunting. An MRI 3 months into therapy showed numerous granulomas, microabscesses and infarcts. Her condition failed to improve with a further course of dexamethasone, and an MRI at 5 months showed increasing size and number of granulomas, with worsening oedema and midline shift (Figure 1, A). She was given a trial of three doses of infliximab $10 \mathrm{mg} / \mathrm{kg}, 1$ month apart, resulting in marked improvement in neurological status and radiological findings (Figure 1, B). She regained movement of her limbs, opened her eyes spontaneously and was able to articulate a few words. After completing 2 months of four-drug therapy, she received isoniazid and rifampicin for 10 months, with ongoing improvement. She was left with mild cognitive deficit and required some assistance with activities of daily living.

\section{Patient 2}

A 32-year-old HIV-negative woman presented with delirium and back pain. A chest radiograph suggested miliary tuberculosis. A computed tomography brain scan was unremarkable. Results of CSF molecular testing were positive for $M$. tuberculosis complex, and cultures from CSF, blood and a laryngeal swab grew fully susceptible M. tuberculosis. Isoniazid, rifampicin, ethambutol, pyrazinamide and prednisolone $50 \mathrm{mg}$ were commenced. One month into therapy, she developed headache in the context of weaning from prednisolone. An MRI showed multiple rimenhancing nodules in the CSF spaces, with leptomeningeal enhancement and enhancing lesions in the right cerebellum and hemipons. CSF cultures tested negative.

The prednisolone dose was increased to $60 \mathrm{mg}$, with little response, then converted to dexamethasone $12 \mathrm{mg} /$ day. One month later, while steroid tapering, she developed diplopia. An MRI showed worsening tuberculomas with increasing oedema (Figure 2, A). Dexamethasone was reinitiated at $12 \mathrm{mg} /$ day. Three months into therapy, she developed obstructive hydrocephalus requiring VP shunting. Over the following weeks, she developed peripheral visual field loss. After 4 months of tuberculosis therapy, a trial of infliximab $5 \mathrm{mg} / \mathrm{kg}$ was initiated. The steroid dose was tapered over the next week without worsening of symptoms, and she was discharged. One month later, an MRI showed moderate improvement (Figure 2, B). Two further doses of infliximab were given over the subsequent 6 weeks,
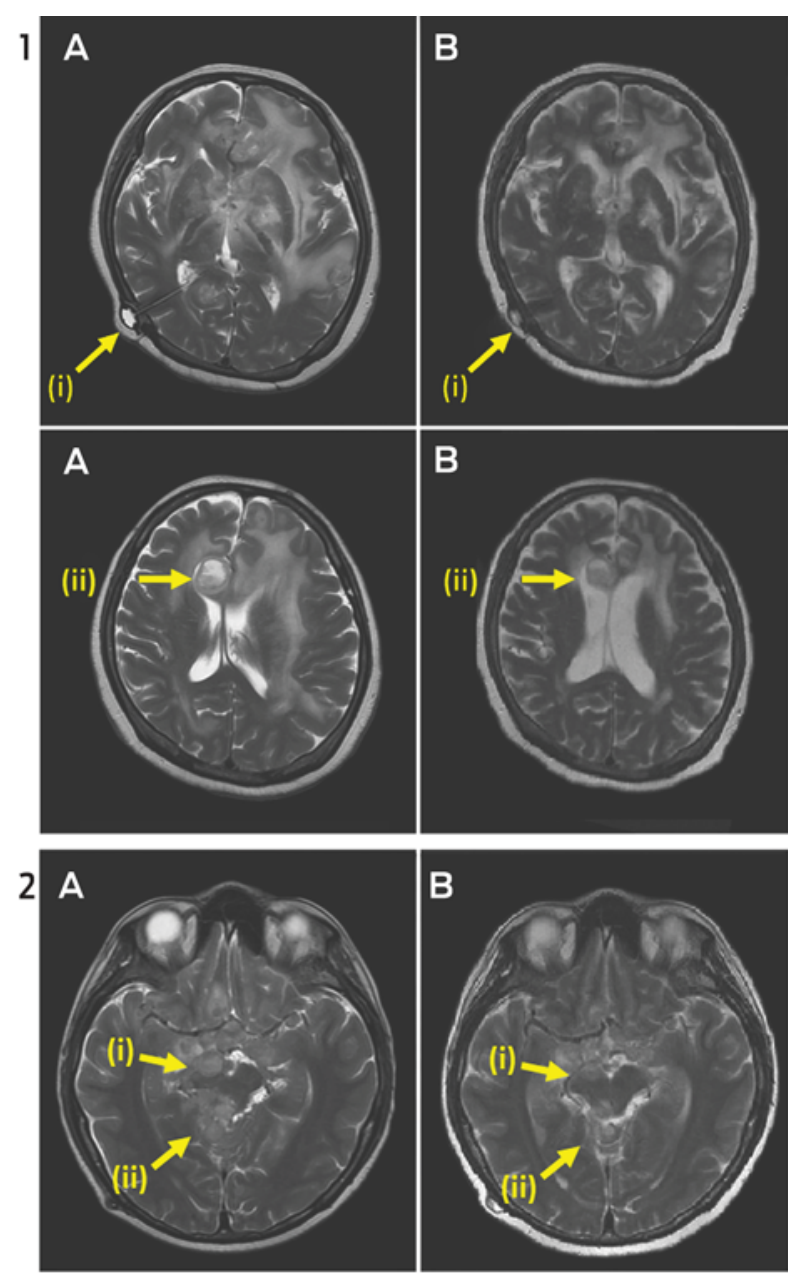

1: Magnetic resonance images (MR/s) for Patient 1 before $(\boldsymbol{A})$ and 3 months after (B) administration of the first dose of infliximab. The first row shows the site of the external ventricular drain placement (i) and the reduction in numerous granulomas throughout. The second row shows the reduction in size of a large tuberculoma (ii) and a decrease in the amount of vasogenic oedema and midline shift.

2: MRIs for Patient 2 before ( $A$ ) and 1 month after (B) administration of the first dose of infliximab, showing reduction in the size and number of granulomas in the right hemipons (i) and superior part of the cerebellum (ii).

with complete resolution of visual symptoms. She completed 2 months of four-drug therapy, followed by 10 months of isoniazid and rifampicin. The course was complicated by a seizure at Month 8, necessitating antiepileptic therapy, but she made an otherwise full neurological recovery.

when tumour necrosis factor alpha (TNF- $\alpha$ ) antagonists are discontinued. ${ }^{3}$ A PR may manifest with new pulmonary lesions or lymphadenopathy ${ }^{3}$ and can be life-threatening, especially in patients with neurotuberculosis. ${ }^{1}$ Management involves high-dose corticosteroids, but in intractable cases success has been reported with TNF- $\alpha$ blockade. ${ }^{1,3-5}$ 


\section{Lessons from practice}

- Central nervous system tuberculosis remains a potentially devastating disease that, despite contemporary therapeutic approaches, still results in permanent disability or death in half of those treated.

- A paradoxical reaction is an inflammatory reaction that can cause disease progression and complications after initiation of antituberculous therapy.

- High-dose corticosteroids are recommended but if these are ineffective, there is mounting evidence for the use of tumour necrosis factor alpha antagonists such as infliximab.

Our two patients had severe neurotuberculosis PRs unresponsive to dexamethasone, which abated after administration of the anti-TNF- $\alpha$ antibody infliximab. Before these two cases, there was only one report of therapeutic use of infliximab for TB PR in an individual without prior history of TNF- $\alpha$ antagonist use. ${ }^{1}$ Our two cases add weight to this approach being safe and effective in patients with steroid-refractory TB PR. The previously reported patient had steroid-refractory neurotuberculosis that did not respond to a trial of cyclophosphamide. Radiological and neurological parameters improved only after infliximab was given. ${ }^{1}$ In that case and ours, cultures tested negative soon after antituberculous therapy was started, suggesting that ongoing disease was due to an immunologically mediated PR rather than inadequate microbiological control.

These cases highlight the potentially devastating effects of central nervous system (CNS) TB, which, despite contemporary therapeutic approaches, still results in permanent disability or death in half of those treated. ${ }^{6}$ Much of this morbidity can be attributed to the inflammatory response. A key inflammatory cytokine is TNF- $\alpha$, which plays an integral role in granuloma formation to contain TB infection. However, in mouse models of neurotuberculosis, TNF- $\alpha$ has been shown to increase blood-brain barrier permeability, resulting in increased CSF leukocytosis and CNS inflammation. ${ }^{7}$

Attenuation of the inflammatory response with routine administration of corticosteroids in patients with neurotuberculosis has been shown to reduce mortality. ${ }^{6}$ However, in cases of PR, outcomes are often poor despite steroids. There is growing evidence that medications with anti-TNF- $\alpha$ activity may have a role in controlling this inflammatory response, without compromising microbiological response. ${ }^{1,3-5}$

Thalidomide, a potent TNF- $\alpha$ inhibitor, was administered to two patients with steroid-refractory neurotuberculosis, with apparent improvement. ${ }^{8}$ While also showing promise in rabbit models and a small pilot study, it was poorly tolerated and failed to show clinical benefit when used as adjunctive therapy for childhood TB meningitis in a randomised trial.

There are accumulating data on the role of the anti-TNF- $\alpha$ monoclonal antibodies infliximab and adalimumab and the soluble TNF- $\alpha$ receptor etanercept. They have potent anti-inflammatory properties and are well tolerated, but have been associated with increased risk of TB in those taking them for autoimmune conditions. ${ }^{10}$ TB developing in patients receiving TNF- $\alpha$ antagonists is more likely to be extrapulmonary or disseminated, ${ }^{11}$ and early reports suggested that it was more refractory to treatment. ${ }^{12}$ However, as experience with these agents grew, it became apparent that the poor response could be a PR to the TNF- $\alpha$ antagonist withdrawal. As the immunosuppressive effect of the TNF- $\alpha$ antagonist wanes, the recovering immune system can generate an intense inflammatory reaction against mycobacterial antigens. Two patients with steroid-refractory disease were successfully treated with reintroduction of the offending TNF- $\alpha$ antagonist. ${ }^{4,5}$

Our report supports an additional role for TNF- $\alpha$ inhibition in severe PRs in immunocompetent individuals. Given that TNF- $\alpha$ antagonists appear to be safe in TB PR, further studies of their role in management are warranted.

Competing interests: No relevant disclosures. 
1 Blackmore TK, Manning L, Taylor WJ, Wallis RS. Therapeutic use of infliximab in tuberculosis to control severe paradoxical reaction of the brain and lymph nodes. Clin Infect Dis 2008; 47 : e83-e85.

2 Pepper DJ, Marais S, Maartens G, et al. Neurologic manifestations of paradoxical tuberculosis-associated immune reconstitution inflammatory syndrome: a case series. Clin Infect Dis 2009; 48: e96-el07.

3 Rivoisy C, Amrouche L, Carcelain G, et al. Paradoxical exacerbation of tuberculosis after TNF antagonist discontinuation: beware of immune reconstitution inflammatory syndrome. Joint Bone Spine 2011; 78: 312-315.

4 Jorge JH, Graciela C, Pablo AP, Luis SH. A life-threatening central nervous system-tuberculosis inflammatory reaction nonresponsive to corticosteroids and successfully controlled by infliximab in a young patient with a variant of juvenile idiopathic arthritis. J Clin Rheumatol 2012; 18: 189-191.

5 Wallis RS, van Vuuren C, Potgieter S. Adalimumab treatment of life-threatening tuberculosis. Clin Infect Dis 2009; 48 : 1429-1432.
6 Thwaites GE, Nguyen DB, Nguyen HD, et al. Dexamethasone for the treatment of tuberculous meningitis in adolescents and adults. N Engl J Med 2004; 351: 1741-1751.

7 Rock RB, Olin M, Baker CA, et al. Central nervous system tuberculosis: pathogenesis and clinical aspects. Clin Microbiol Rev 2008; 21: 243-261.

8 Roberts MT, Mendelson M, Meyer P, et al. The use of thalidomide in the treatment of intracranial tuberculomas in adults: two case reports. J Infect 2003; 47: 251-255.

9 Schoeman JF, Springer P, van Rensburg AJ, et al. Adjunctive thalidomide therapy for childhood tuberculous meningitis: results of a randomized study. J Child Neurol 2004; 19: 250-257.

10 Wallis RS. Reconsidering adjuvant immunotherapy for tuberculosis. Clin Infect Dis 2005; 41: 201-208.

1 Harris J, Keane J. How tumour necrosis factor blockers interfere with tuberculosis immunity. Clin Exp Immunol 2010; 161: 1-9.

12 Vlachaki E, Psathakis K, Tsintiris K, Iliopoulos A. Delayed response to anti-tuberculosis treatment in a patient on infliximab. Respir Med 2005; 99: 648-652. 